\title{
CONOCIMIENTO DE LOS ESTUDIANTES DE LA FACULTAD DE ENFERMERÍA DE LA UNIVERSIDAD NACIONAL "SAN LUÍS GONZAGA" DE ICA SOBRE DETECCIÓN PRECOZ DE CÁNCER DE MAMA. OCTUBRE 2009 - SETIEMBRE 2010
}

\author{
Knowledge of students from the Faculty of Nursing National University "San Luis Gonzaga" of Ica \\ on early detection of breast cancer. October 2009 - September 2010
}

Isabel Natividad Urure Velazco ${ }^{1, a, d}$, Cecilia Teresa Ventura Miranda ${ }^{2, a, b}$, Gladys Marcelina Puza Mendoza $a^{3, a, c}$

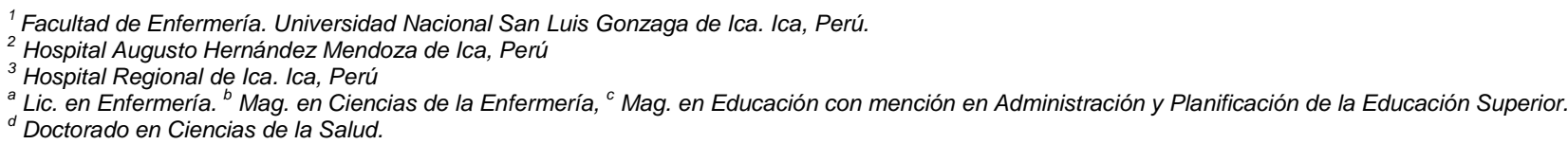

\section{RESUMEN}

Objetivo: Determinar el nivel de conocimiento que tienen los estudiantes de la Facultad de Enfermería de la Universidad Nacional "San Luís Gonzaga" de Ica, sobre detección precoz de cáncer de mama. Material y métodos: Diseño descriptivo transversal. Muestra constituida por 136 estudiantes del I al VIII ciclo, cuestionario validado con preguntas cerradas dicotómicas y de opción múltiple. Se utilizó el coeficiente de confiabilidad Alfa-Cronbach, obteniéndose un valor de 0,75 , así como la prueba de chi cuadrado $\left(\mathrm{x}^{2}\right)$ para determinar significancia entre los indicadores y los ciclos de estudios. Resultados: Predomino sexo femenino $92,6 \%$. Conocimiento de la práctica de autoexamen de mama: $72,8 \%$, frecuencia mensual de práctica $61,8 \%$ desconocen momento pos menstrual de realizarlo $78,7 \%$, conoce otros medios de diagnóstico 76,5\% (Mamografía, TAC), conoce medio de diagnóstico definitivo (Biopsia) 23,5\%, conoce edad más frecuente de aparición 71,3\% (40 a 50 años), y factores de riesgo: (edad y sexo) $94,1 \%$, fuente de información fue la difusión masiva $44,1 \%$. Prueba estadística de chi cuadrado $\left(x^{2}\right)$, se encontró significancia entre frecuencia, momento, edad más frecuente de aparición, medios de diagnóstico, principal fuente de información, y los ciclos de estudios $\left(x^{2}\right) \mathrm{P}<0,01$ los dos primeros y $\left(x^{2}\right)$ $P<0,05$ los tres siguientes. No encontrándose significancia: factores de riesgo (edad y sexo), antecedentes de práctica de autoexamen de mamas, posición para autoexamen y los ciclos de estudios: $\left(x^{2}\right) P>0,05$. Conclusiones: Existe conocimiento medio de los estudiantes de enfermería sobre detección precoz de cáncer de mama, con promedio porcentual de respuestas correctas $69,35 \%$.

Palabras Clave: Conocimiento, estudiantes enfermería, autoexamen de mamas.

\section{SUMMARY}

Objective: Determine the level of knowledge among students of the Faculty of Nursing at the National University "San Luis Gonzaga" of Ica, about early detection of breast cancer. Material and Methods. Cross-sectional design. Sample consisting of 136 students from I to VIII cycle validated questionnaire with closed questions and multiple-choice dichotomous. The reliability coefficient Cronbach Alpha was used to give a value of 0,75 , and the chi-square test $\left(x^{2}\right)$ to determine significance between indicators and courses of study. Results: $92,6 \%$ female predominance. Knowledge of the practice of breast selfexamination: $72,8 \%$, monthly practice often unaware $61,8 \%, 78,7 \%$ postmenstrual of doing currently known other diagnostic means 76,5\% (Mammography, CT), half knows definitive diagnosis (biopsy) $23,5 \%$, knows most common age of onset $71,3 \%$ (40-50 years), and risk factors (age and sex) $94,1 \%$, source of information was the mass dissemination $44,1 \%$. Statistical chi square test $\left(x^{2}\right)$, significance of frequency, time, most common age of onset, diagnostics, the main source of information and study cycles $\left(x^{2}\right) P<0,01$ the first two met and ( $\left.x 2\right) P<0,05$ the following three. Not finding significance: risk factors (age and sex), history of breast self-examination practice, position and self study cycles $\left(x^{2}\right) P>$ 0,05 . Conclusions: There average knowledge of nursing students on early detection of breast cancer, with average percentage of correct responses $69,35 \%$.

Keywords: Knowledge, nursing students, breast self-examination. 


\section{INTRODUCCIÓN.}

El cáncer de mama es la neoplasia más frecuente en la mujer susceptible de diagnóstico y tratarse precozmente, obteniéndose así excelentes resultados con un período de vida larga y libre de enfermedad. Sin embargo la realidad es otra. Constituye un serio problema de salud en el mundo (1). Se estima que una de cada 10 mujeres estadounidenses desarrolla cáncer mamario durante el transcurso de su vida (2). Según la sociedad Estadounidense del cáncer, los tumores mamarios son la principal causa de muerte por cáncer entre las mujeres en todo el mundo (1). En países de América Latina se han encontrado tasas de $65,5 \%$ por 100,000 mujeres en 1978 en Sao Paulo, Brasil y 35,1\% en 1980 en Puerto Rico (3). Es un grave problema de salud pública en la región de América Latina y el Caribe (LAC). Es hoy a diferencia de hace 15 años una de las enfermedades con mayor incidencia en la mujer adulta en América Latina y el Caribe (1). Los pacientes en más del $50 \%$ acceden a la primera consulta con enfermedad avanzada; lo cual hace que su tratamiento sea más complicado y caro, obteniéndose supervivencia corta y con enfermedad evolutiva (4).

La revisión bibliográfica de unos autores plantea que existe una deficiente educación médica de la población general, atribuyéndosele la responsabilidad a los médicos y las instituciones de salud de los países $(2,4)$. La mayoría de las muertes por cáncer de mama pueden evitarse. La evidencia internacional señala que es curable en más del $95 \%$ de los casos, si se detecta precozmente la enfermedad es localizada y el tratamiento médico es el adecuado (5). En México un estudio de la secretaria de salud revela que hasta dos terceras partes de los fallecimientos en mujeres menores de 75 años por cáncer de mama se pueden evitar con detección temprana de la enfermedad (6). Argentina es uno de los países con más casos de cáncer de mama en el mundo. Se calcula que una de cada 8 mujeres podría desarrollar la enfermedad en algún momento de sus vidas (7). En Cuba las tasas de morbilidad y mortalidad del cáncer de mama han aumentado considerablemente en los últimos años. El cáncer de mama es la localización más frecuente del cáncer entre las neoplasias malignas de la mujer y representa la primera causa de muerte por cáncer en la mujer (8). De la misma manera en nuestro país (Perú) el cáncer de mama es el tumor que mayor número de muertes produce en la mujer y también la primera causa de muerte entre 40 y 55 años.

$\mathrm{Su}$ incidencia esta pues en aumento sobre todo en los países desarrollados. A pesar de que aumenta la incidencia, la tasa de mortalidad ha disminuido en los últimos años, estos beneficios se atribuyen a los programas de detección precoz y a los avances en el tratamiento sistémico (Levi 2005). Es decir que las estadísticas alarmantes en EE. UU. obligan a las enfermeras a ver este diagnóstico teniendo en mente prevención, enseñanza y cuidados. La frecuencia del cáncer mamario aumenta en tanto que la proporción de muertes permanece casi igual. La enfermedad puede ocurrir a cualquier edad después de iniciarse la menstruación, pero es más frecuente a partir de los 40 años (9). Se observó el incremento de riesgo en mujeres mayores de 40 años, y otros factores de riesgo como: Histórico de la enfermedad, familiar o de familia próxima, en multíparas, primer embarazo después de los 30 años. Dado que los estudios actuales muestran una mayor conexión entre estrógenos y cáncer de mama la menarquía precoz y tardía se añaden actualmente como factores de riesgo (10).

Considerando que la detección precoz es la clave del tratamiento con éxito del cáncer de mama así como la mejor vacuna contra el cáncer es la prevención. $Y$ que el conocimiento (saber, sabiduría) de los medios de diagnóstico para detectar precozmente el cáncer de mama disminuye los índices de mortalidad, como el auto examen de mamas poco costosa y que en nuestra cultura una proporción significativa se encuentran ellas mismas la masa como consecuencia de un examen deliberado, de una palpación accidental mientras se bañan o en otra circunstancia, se debe hacer énfasis en la paciente su instrucción aunque es impreciso. 
Así como otros medios de diagnóstico conocidos: mamografía, ultrasonido, etc. Pero el diagnóstico definitivo del cáncer mamario solo se puede esclarecer de manera histológica (biopsia abierta). En este sentido, las enfermeras y/o estudiantes de enfermería tienen una función importante en la reducción de los tumores malignos mamarios a través de la investigación, asistencia a la paciente y compromiso para la enseñanza y prevención. La participación de la enfermera en la enseñanza del auto examen de las mamas a todas las pacientes es la clave para disminuir la proporción de mortalidad por esta enfermedad. El presente estudio tuvo como objetivo Determinar el nivel de conocimiento que tienen los estudiantes de la Facultad de Enfermería de la Universidad Nacional "San Luís Gonzaga" de Ica, sobre detección precoz de cáncer de mama.

\section{MATERIAL Y MÉTODOS.}

Se realizó un estudio descriptivo de corte transversal entre octubre 2009 y octubre 2010 en los estudiantes de la Facultad de Enfermería de la Universidad Nacional San Luis Gonzaga de Ica, siendo área de estudio ubicada en la Av. Los Maestros Ciudad Universitaria Km. 305, en el departamento y provincia de Ica, país Perú. La muestra lo constituyo 136 estudiantes del I al VIII Ciclo $\left(1^{\circ}\right.$ al $4^{\circ}$ Año). Se empleó una fórmula para la determinación del tamaño de la muestra, haciendo uso de un cuestionario de 10 preguntas cerradas dicotómicas y opción múltiple. El cuestionario estuvo conformado por 2 partes: Primera parte: Datos generales (edad, sexo, año o ciclo de estudios) y la Segunda Parte: Acerca de los conocimientos de los estudiantes de la Facultad de Enfermería, sobre detección precoz de cáncer de mama. Se excluyó estudiantes que se encuentran realizando el internado (prácticas pre-profesionales) en las diferentes instituciones de salud. En la recolección de datos se utilizó como técnica la encuesta y como instrumento el cuestionario previamente validado con preguntas cerradas. Para evaluar la confiabilidad del instrumento se realizó la prueba piloto al $15 \%$ de la muestra en estudio (no se incluye los 136 estudiantes) que permitieron determinar las características finales del instrumento, utilizando el coeficiente de confiabilidad alfa-cronbach, obteniendo un valor de 0,75. Así como la prueba estadística del Chi cuadrado $\left(x^{2}\right)$ para determinar significancia 0 no entre los indicadores y los ciclos de estudios. Se hizo uso de un programa de análisis de datos tabulados (EPIDAT 2.1).

\section{RESULTADOS.}

Edad mínima 17 años y máxima 27 años. Sexo predominante el femenino 92,6\%. (Tabla 1) Se observó un conocimiento de los estudiantes respecto a la edad que se presenta con mayor frecuencia el cáncer de mama que es entre 40 y 50 años el $71,3 \%$. Como factores de riesgo señalaron la edad y sexo $94,1 \%$. Como fuente de información es mayoritariamente la difusión masiva con $44,1 \%$. Que la participación de la enfermera en enseñanza del auto-examen de mama clave para disminuir la proporción de mortalidad por esta enfermedad respondieron el 96,3\%. La exploración minuciosa de la mama es fundamental en la detección precoz del cáncer de mama, respondieron mayoritariamente el 94,9\%. Conocimiento de la práctica del auto-examen de mama el $72,8 \%$. Respecto a la frecuencia mensual de práctica del auto-examen de mama, posición (Decúbito dorsal, sentado o de pie) y momento post mensual de efectuarse, respondieron: el $61,8 \%, 88,2 \%$ y $21,3 \%$ respectivamente.

El medio de diagnóstico definitivo (biopsia) para determinar el diagnóstico de cáncer de mama, solo el $23,5 \%$ respondieron correctamente (Tabla 2). En la prueba estadística de chi cuadrado $\left(x^{2}\right)$, frecuencia, momento, edad que se presenta con mayor frecuencia, medios de diagnóstico, principal fuente de información y los ciclos de estudios se encontró $\left(x^{2}\right) p<0,01$ los 2 primeros y $\left(x^{2}\right)$ $p<0,05$, los tres siguientes. No encontrándose significancia factores de riesgo (edad y sexo), antecedentes de práctica, posición, participación de la enfermera en la enseñanza del autoexamen de mamas y exploración mamaria minuciosa fundamental para detección precozmente el cáncer de mamas y los ciclos de estudios $p>0,05$. 
TABLA 1. CONOCIMIENTO DE LOS ESTUDIANTES DE LA FACULTAD DE ENFERMERÍA DE LA U.N. "SAN LUIS GONZAGA” DE ICA SOBRE DETECCIÓN PRECOZ DEL CÁNCER DE MAMA. OCTUBRE 2009 - SETIEMBRE 2010, POR EDAD SEGÚN SEXO.

\begin{tabular}{ccccccc}
\hline & \multicolumn{4}{c}{ SEXO } & \multicolumn{2}{c}{ TOTAL } \\
\cline { 2 - 5 } EDAD & \multicolumn{3}{c}{ Masculino } & \multicolumn{2}{c}{ Femenino } & \multicolumn{1}{c}{ Tet } \\
\cline { 2 - 5 } & № & $\%$ & № & $\%$ & № & $\%$ \\
17 & 1 & 0,7 & 6 & 4,4 & 7 & 5,1 \\
18 & 2 & 1,5 & 21 & 15,4 & 23 & 16,9 \\
19 & 1 & 0,7 & 30 & 22,1 & 31 & 18,4 \\
20 & 1 & 0,7 & 22 & 16,2 & 23 & 16,9 \\
21 & 4 & 2,9 & 21 & 15,4 & 25 & 18,4 \\
22 & 1 & 0,7 & 13 & 9,6 & 14 & 10,3 \\
23 & 0 & 0,0 & 5 & 2,7 & 5 & 2,7 \\
24 & 0 & 0,0 & 4 & 2,9 & 4 & 2,9 \\
25 & 0 & 0,0 & 1 & 0,7 & 1 & 0,7 \\
26 & 0 & 0,0 & 1 & 0,7 & 1 & 0,7 \\
27 & 0 & 0,0 & 2 & 1,5 & 2 & 1,5 \\
\hline TOTAL & 10 & 7,4 & 126 & 92,6 & 136 & 100,0 \\
\hline
\end{tabular}

TABLA 2. RESPUESTAS CORRECTAS DE LOS ESTUDIANTES DE LA FACULTAD DE ENFERMERÍA DE LA U.N. "SAN LUIS GONZAGA" DE ICA DEL CUESTIONARIO DE PREGUNTAS APLICADO.

\begin{tabular}{|c|c|}
\hline PREGUNTA & $\begin{array}{l}\text { RESPUESTA } \\
\text { CORRECTA (\%) }\end{array}$ \\
\hline $\begin{array}{l}\text { 1. A qué edad se presenta con mayor frecuencia cáncer } \\
\text { de mama }\end{array}$ & 71.3 \\
\hline $\begin{array}{l}\text { 2. Los factores de riesgo más importante para el cáncer } \\
\text { de mama son el sexo femenino y la edad }\end{array}$ & 94,1 \\
\hline $\begin{array}{l}\text { 3. Cree usted que la participación de la Enfermera en la } \\
\text { enseñanza del auto-examen de las mamas a todos los } \\
\text { pacientes es la clave para disminuir la proporción de } \\
\text { mortalidad por esta enfermedad }\end{array}$ & 96,3 \\
\hline $\begin{array}{l}\text { 4. La exploración mamaria minuciosa es fundamental para } \\
\text { la detección precoz del cáncer de mama? }\end{array}$ & 94,9 \\
\hline $\begin{array}{l}\text { 5. Tiene conocimiento de cómo se practica el auto- } \\
\text { examen de mama }\end{array}$ & 72,8 \\
\hline $\begin{array}{l}\text { 6. Con qué frecuencia debe practicarse el auto-examen de } \\
\text { mama }\end{array}$ & 61,8 \\
\hline $\begin{array}{l}\text { 7. Momento del ciclo menstrual en que se debe realizar el } \\
\text { auto -examen de mama }\end{array}$ & 21,3 \\
\hline $\begin{array}{l}\text { 8. En cuanto a la posición para la exploración de mamas } \\
\text { es: de cúbito dorsal, de pie o sentada frente a un espejo }\end{array}$ & 88,2 \\
\hline $\begin{array}{l}\text { 9. Cuál de los medios de diagnóstico va a determinar el } \\
\text { diagnóstico definitivo de cáncer de mama }\end{array}$ & 23,5 \\
\hline $\begin{array}{l}\text { PROMEDIO PORCENTUAL DE RESPUESTAS } \\
\text { CORRECTAS }\end{array}$ & 69,35 \\
\hline
\end{tabular}

\section{DISCUSIÒN.}

La mama constituye la principal localización de cáncer de la mujer, susceptible de diagnóstico y tratarse precozmente, mediante una detección precoz, siendo importante la instrucción de la práctica del auto examen de mama especialmente en la mujer. Este estudio mostró un predominio del sexo femenino 92,6 siendo este sexo considerado como factor de riesgo porque refiere la literatura que la mayoría de las mujeres no tiene factores de riesgo 
discernibles, pero están en peligro debido a su sexo, por lo que se le debe evaluar con cuidado (9), de ahí la importancia de un conocimiento adecuado de una detección precoz del cáncer de mama. Valores similares hallados en la ciudad de la Habana por Segura N. et al (11) representado el 68,5. Referente a la edad que se presenta con mayor frecuencia el cáncer de mama considerando que el cáncer de mama es el tumor que mayor número de muertes produce en la mujer en nuestro país y la primera causa de muerte en mujeres entre los 40 y 55 años. El estudio mostró sólo un 28,7 desconocimiento de la edad que se presenta con mayor frecuencia el cáncer de mama. Como se conoce, la enfermedad aparece a partir de los 40 años y aumenta las probabilidades de la misma a medida que la edad avanza (9). En cuanto a los factores de riesgo más importantes del cáncer mamario, es el sexo femenino y la edad, siendo más frecuente a partir de los 40 años. Aquí mostró la tabla un conocimiento con 94,1 lo que revela que a pesar de que aumenta la incidencia, la tasa de mortalidad, ha disminuido en los últimos años. Estos beneficios se atribuyen a los programas de detección precoz y a los avances en el tratamiento sistémico (Leví 2005), siendo la principal fuente de adquisición de los conocimientos la difusión masiva $(44,1 \%)$, similares resultados en publicaciones revisadas reporto Segura N et al (11), en la ciudad de la Habana representando un $51 \%$. Dado que el cáncer de mama constituyó la segunda causa de muerte por este tipo de enfermedad entre la población femenina $y, \quad$ sin embargo detectado a tiempo puede ser eficazmente tratado (12). El 95 de los casos atendidos a tiempo tienen cura (13), a través de una detección temprana del cáncer de mama, mediante la exploración mamaria minuciosa, corroborando el estudio de investigación con la literatura que respondieron el 94,9 correctamente. En cuanto al conocimiento de los antecedentes del autoexamen de mama, la muestra señaló un 27,2, no poseen conocimiento íntegro de la práctica del auto-examen de mamas. Siendo importante el conocimiento del mismo adecuado y correcto que permitirá una detección temprana del cáncer de mama. Similares resultados en publicaciones de Chapoñan et al (14), señaló que el 29,3 no se examinan sus mamas. En cuanto al conocimiento de la frecuencia que debe practicarse el autoexamen de mama, el 29,3 del total de los pacientes encuestados no poseen conocimiento de la frecuencia con que debe practicarse el autoexamen de mamas, la mujer ha de conocer que es conveniente realizar la exploración mamaria todos los meses. Hay que instruir a los pacientes para que se continúen haciéndolo cada mes después de la menopausia, ya que la incidencia de esta neoplasia se incrementa de manera dramática en el climaterio (2). El 78,7 del total de estudiantes encuestados no tienen conocimiento del momento del ciclo menstrual en que debe realizarse el autoexamen de mama. Debiendo realizarse post-menstrual o sea después de la menstruación en el cual las mamas no están hinchadas ni doloridas 0 si es irregular en un día preciso. En cuanto a la posición para la exploración de mamas decúbito dorsal, de pie o sentada, sólo un 11,8 del total de estudiantes encuestados no poseen conocimiento que es decúbito dorsal, de pie o sentada, lo cual es relevante que un gran porcentaje $(88,2)$ conoce las posiciones adecuadas para una exploración minuciosa con buena iluminación conducentes a una detección eficaz del cáncer de mama. La mayoría de los estudiantes encuestados conoce otros medios de diagnósticos (76,5 Mamografía, Tomografía Axial Computarizada TAC), no identificando la biopsia como medio de diagnóstico definitivo, el que solo puede establecerse de manera histológica, señalando acertadamente el 23,5. 


\section{CONCLUSIONES.}

1. Los estudiantes de la Facultad de Enfermería tienen conocimiento del autoexamen de mama en la detección precoz del cáncer de mama respecto del antecedente como se practica el $72,8 \%$. Conoce la frecuencia mensual de práctica el $61,8 \%$. En cuanto a la posición, conocen gran porcentaje el $88,2 \%$. Desconocen momento del ciclo pos menstrual de realizarlo el $78,7 \%$. Conoce el $21,3 \%$. Conoce otros medios de diagnóstico el $76,5 \%$ (Mamografía, TAC) pero conoce como medio de diagnóstico definitivo (Biopsia) el 23,5\%.

En la prueba estadística del Chi Cuadrado $\left(x^{2}\right)$ se encontró que existe diferencia significativa $(P<0,01)$ en la frecuencia, momento y $(P<0,05)$ en el medio de diagnóstico y los ciclos de estudios.

2. En cuanto a la edad (40-50) más frecuente de aparición conoce el $71,3 \%$ y conoce como factores de riesgo la edad y sexo el $94,1 \%$. En la prueba estadística de chi cuadrado $\left(x^{2}\right)$ se encontró que existe diferencia significativa $(P<0,05)$ entre los grupos de edad, no encontrándose diferencia significativa $(P>0,05)$ en factores de riesgo (edad y sexo) y los ciclos de estudios.

3. La principal fuente de adquisición de información de conocimientos acerca de la detección precoz del cáncer de mama, fue los medios de difusión masiva con un $44,1 \%$. En la prueba estadística de chi cuadrado $\left(x^{2}\right)$ se encontró que existe diferencia significativa $(P<0,05)$ entre fuente de adquisición de información y los ciclos de estudios.

4. Se observó un predominio del sexo femenino $92,6 \%$.

\section{CONCLUSIÓN FINAL.}

Se concluye un conocimiento medio de los estudiantes de Enfermería sobre detección precoz del cáncer de mama con un promedio porcentual de respuestas correctas de $69,35 \%$

\section{RECOMENDACIONES.}

Que el profesional de Enfermería docentes y asistenciales difundan a todo nivel (primario, secundario y superior) como medida preventiva-promocional, conocimientos sobre detección precoz del cáncer de mamas, para fortalecer e incrementar el porcentaje de los mismos, contribuyendo con ello a disminuir los índices de morbimortalidad.

\section{Correspondencia:}

Dra. Isabel Natividad Urure Velazco

Correo electrónico: isabelnauv@yahoo.com

\section{REFERENCIAS BIBLIOGRÁFICAS.}

1. Lozano R., Gomez D, Arreola H., Méndez Q. y Knaul, F.M. 2008 El Cáncer de Mama en México. Cifras para la toma de decisiones. Observatorio de la Salud. Documento de trabajo Competitividad y Salud. Fundación Mexicana para la Salud. (Internet). México 2009. ( Citado el 12 de Febrero 2010) Disponible en: http://200.57.141 .184/docum/ hoja \%20datos cama.pdf

2. Norman F. Gant, F. Gary Gunninghan. Manual Gineco Obstetrico. México: Ed. El Manual Moderno S.A.: 1998: Cap. 14, p. 134.

3. Botero J., Jubiz A. Obstetricia y Ginecología. 6aㅡ ed. Bogotá Colombia: Ed. Fondo de la Cib: 2001: Cap. 53.

4. Urquizo A. Cáncer de Mama. ( Internet) México: 2009 (Citado el 9 de Enero 2010) Disponible en: http://sisbib.unmsm.edu.pe

5. American Cáncer Society 2008 "Breast Cancer Facts, Figurs 2007-2008”. Atlanta American Cancer Society In (Internet) EE.UU.2008 (Citado el 2 de Febrero 2010) Disponible en: http://www.cancer.org.

6. Franco M., Lozano B., Vella P., Solis La Mortalidad en México 2000-2004 Muertes evitables: Magnitud, distribución y tendencias". Dirección General de Información en Salud. Secretaría de Salud (Internet) México: 2006 http://www.sisbib.unmsm.edu.pe [Consulta setiembre 2009]. 
7. Pérez A. Ginecología $2^{a}$ ed. Santiago de Chile: Ed. Mediterráneo: 1995 p. 359-360.

8. Ministerio de Salud Pública (MINSAP) Anuario Estadístico de Salud año 2007. La Habana Cuba. MINSAP: 2007 p. 109- 112.

9. Brunner y Suddarth. Enfermería Médico Quirúrgica. México: Ed. Interamericana Mc. Graw-Hill: 1997: Cap. 46 p. 1367.

10. Dickason E., Silverman B. Schultt $M$. Enfermería Materno Infantil. $2^{\mathrm{a}}$ ed. Barcelona, España: Ed. Harcout Brace: 1997: Cap. 4 p. 68.

11. Segura N, Mulen S, Medina I, Gil y Ferrás M. Conocimiento sobre detección precoz del cáncer de mama en estudiantes del primer año de Ciencias Médicas en ciudad de La Habana .Tesis Medicina Preventiva en Salud Pública. Habana,Cuba; 2009.

12. LEXUS. Manual de Enfermería. Barcelona España: Ed. Lexus editores: 2003: p. 565566.
13. Periódico Comercio: Lima, Perú 14 de Setiembre del 2009.

14. Chapoñan, Quispe y Rojas. Detección precoz del cáncer mamario .Tesis para optar el título de Licenciado en Enfermería .Lima, Perú Universidad San Martin de Porres (U.S.M.P.) 1997.

Recibido: 12/11/2014

Aprobado para publicación: 28/04/2015 